\title{
Leela Quantum Bloc Shows Protective Effects on the Blood Upon Human Exposure to Short-Term Wi-Fi
}

\author{
Pilot Study Report \\ Beverly Rubik, Ph.D. \\ January, 2022
}

\begin{abstract}
Blood is the essence of life and is frequently monitored in health and wellness testing. In this pilot study, twelve healthy human subjects were exposed to pulse-modulated microwave radiation from an idle $\mathrm{Wi}-\mathrm{Fi}$ router situated at 2 meters from them. This was a randomized, single-blinded, sham-controlled trial to look for a protective effect on the blood from the use of Quantum Bloc. Fresh blood samples from finger pricks were examined under a dark-field microscope at baseline (no exposure); after Wi-Fi exposure for 10 minutes; and again with Wi-Fi exposure for another10 minutes with either a sham or a Quantum Bloc, during which time each subject's hands were placed inside the cube. Blood photos were scored using Likert scales for morphological factors, and the results were analyzed for statistical differences using paired ttests. The blood showed significant adverse changes following 10 minutes of subject exposure to Wi-Fi radiation: (1) increased rouleaux $\left(\mathrm{p}=9 \times 10^{-13}\right)$; (2) increased generalized red blood cell aggregation $\left(\mathrm{p}=5 \times 10^{-12}\right)$; reduced white blood cell motility $(\mathrm{p}=0.002)$; increased early fibrin $(\mathrm{p}=0.001)$; and increased misshapen red blood cells $(\mathrm{p}=0.003)$. Exposure to the Quantum Bloc compared to the sham device showed statistically significant protective effects on the blood, beyond the effects of the sham: (1) reduced rouleax $(p=0.0004) ;(2)$ reduced
\end{abstract}


generalized red blood cell aggregation $(\mathrm{p}=0.001),(3)$ increased white blood cell motility $(\mathrm{p}=$ 0.003); and reduced early fibrin $(\mathrm{p}=0.04)$. Quantum Bloc impacted the blood of all subjects positively, and furthermore, positively impacted all readily observable adverse effects of the radiation on the blood. In conclusion, human exposure to radiation from a Wi-Fi router produced adverse changes in the morphology of their blood that are clearly observed using an optical microscope. Quantum Bloc showed a robust protective effect against these adverse blood changes.

\section{RESEARCH QUESTIONS}

1. Does the blood as observed under a dark-field microscope change after human subjects are exposed to radiation emitted from a Wi-Fi router in idle mode for 10 minutes?

2. Does a Quantum Bloc help protect human subjects from adverse blood changes seen upon exposure to radiation from a Wi-Fi router significantly compared to a sham device?

\section{INTRODUCTION AND RESEARCH DESIGN}

In this study, live blood was examined and photographed under a microscope to look for morphological changes in reaction to a stressor, wireless communication radiation emitted by a Wi-Fi router. Previously an exploratory pilot study with four healthy human subjects was 
conducted to look for a protective effect of the Quantum Bloc device compared to sham. As in the present study, peripheral blood samples taken from each subject's fingertips were placed on glass slides observed under a dark-field microscope, photographed, and scored by a trained blood research microscopist using a Likert scale ( 0 to 4 , with 4 being the maximum). Data analysis showed that the Quantum Bloc appeared to protect the blood. However, the sample size ( $N=4)$ was too small for statistical analysis.

In the present study, the same techniques were used on twelve healthy adults, with statistical analysis of the data. This study was a randomized, sham-controlled, single-blinded study in which subjects were blinded as to the identity of the sham or active device.

\section{SUBJECTS}

Subjects were healthy adults consisting of 3 males and 9 females ranging from 42 to 80 years of age, with a mean age of 62.25 years (See Figure 1). Subjects, all Caucasians and Asians, included a diversity of ethnicities. They were recruited by word-of-mouth in the San Francisco Bay Area. A middle-to-elderly population was selected because a previous study showed that older adults had greater adverse blood changes from exposure to wireless radiation (Rubik, 2014). None of the subjects had a diagnosis of electrosensitivity.

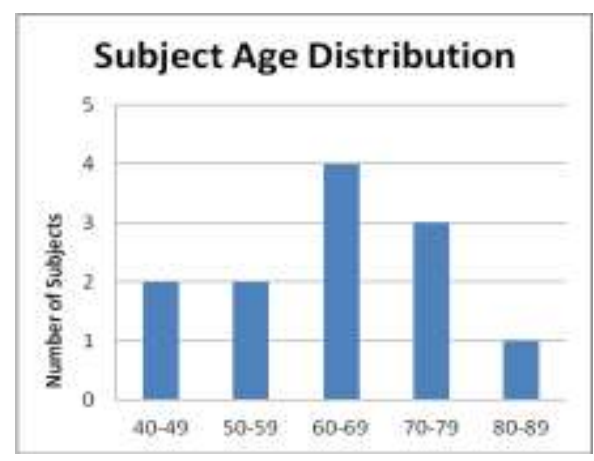

Figure 1. Subject Age Distribution 


\section{PROCEDURES}

The wireless router used for subject exposure was a Linksys model EA7500 with 3 antennas emitting radiofrequencies at 2.4 and $5.0 \mathrm{GHz}$, modulated at $10 \mathrm{~Hz}$. It was used in idle mode--not connected to a computer--so that it could neither download nor upload data, and produced the same emission pattern consistently over time. Using an oscilloscope in the time domain, the emission pattern is shown in Figure 2. The power density to which subjects were exposed for 10 minutes was $-25 \mathrm{dBm}$ (decibels) or $1.8 \mathrm{~mW} / \mathrm{m}^{2}$. By comparison, the USA guideline set by the Federal Communication Commission (FCC) for maximum public exposure is $+10 \mathrm{dBm}$ or $5.8 \mathrm{~W} / \mathrm{m}^{2}$.

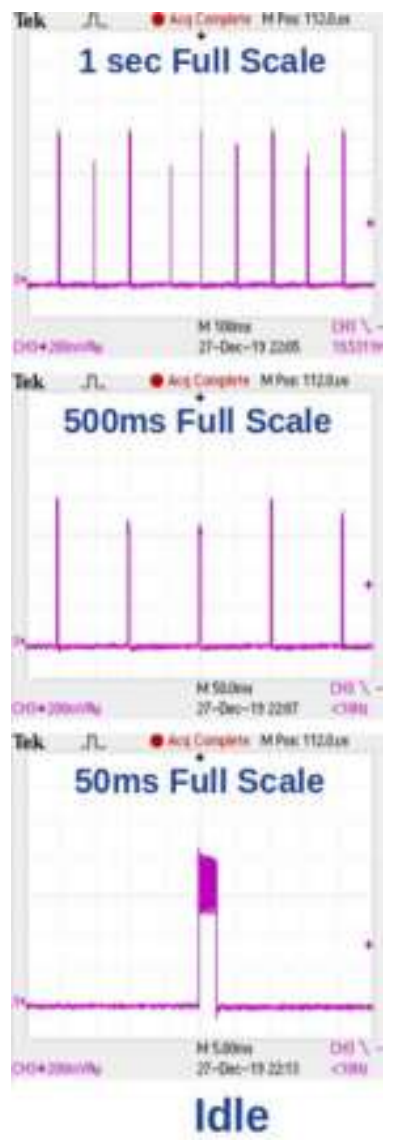

Figure 2. Wi-Fi Router in idle mode, showing the $10 \mathrm{~Hz}$ digitally pulsed modulation on oscilloscope trace. 
Live blood analysis involves examination of a small droplet of fresh capillary blood typically taken from the fingertip of human subjects. Typically an optical microscope at magnifications from 600 to $1200 x$ is employed. In this study, a custom-built, dark-field microscope attached to a digital video camera system with zoom lens linked to a computer monitor was used. The blood specimen was lit by means of light delivered through fiber optics attached to the microscope condenser to prevent sample heating. Oil immersion lenses at the microscope objective and dark-field condenser were used for image optimization. A camera mounted on the microscope recorded digital photographs of the blood samples. This technique provides information on the ecology of the blood, sometimes referred to as the "biological terrain," also used in holistic health assessment. The size, shape, variability, and cellular integrity of the red blood cells (RBCs) can readily be seen, as well as any stickiness and aggregation of the RBCs. The presence of white blood cells (WBCs) is noted along with the motility (movement) of these cells. The blood plasma was also assessed for the formation of early fibrin $(<10$ minutes).

The study was conducted in fall and winter of 2021. Subjects fasted for at least 5 hours and refrained from use of their wireless communication devices for at least one hour prior to individual appointments. They were tested on different days at approximately the same time of day, with the presentation of the sham device and Quantum Bloc in a randomized order. The sham and Quantum Bloc devices appeared to be the same except for the presence of a flower pattern on the plates of the active device. During the fasting period and the experimental session, subjects were allowed to drink only water. Each subject was given 3 blood tests associated with 3 different exposure conditions as described below. A sterile lancet was used to collect a droplet 
of peripheral blood from each of 3 fingertips, which was immediately placed on a glass microscope slide covered with a glass cover slip, and observed and photographed for 10 minutes.

Each blood sample was evaluated and scored for the various blood morphological factors by the researcher, who is also an experienced blood microscopist. The factors include the shape of red blood cells with respect to any distortion (membrane distortion); state of aggregation of the RBCs, including non-specific clumping as well as rouleaux formation (cells stuck together in rolls); WBC motility; and the level of early fibrin which is indicative of blood clotting. A Likert scale from 0 to 4 in increments of 0.5 was used to score the blood factors, in which 0 indicates the absence of the blood factor, and 4 indicates the maximum observed level of the blood factor.

Three blood tests were performed on each subject as follows: (1) initially, prior to Wi-Fi exposure ("baseline"), for which the radiofrequency exposure was $-42 \mathrm{dBm}$, the ambient level in the laboratory; (2) following 10 minutes of exposure to Wi-Fi from a router placed 2 meters from the subject, during which the radiofrequency exposure was $-25 \mathrm{dBm}$ ("post-exp"); and (3) following an additional 10 minutes of exposure to the $-25 \mathrm{dBm}$ level of Wi-Fi while placing their hands inside either the sham or active device ("post exp \& device"). At least six typical blood microphotographs were made for each of the 3 exposure conditions for each subject.

\section{RESULTS}

Hundreds of blood microphotographs and an Excel file of compiled data, calculations, and graphs accompany this report. Figure 3 shows sample blood photographs illustrating (a) typical healthy blood prior to Wi-Fi exposure; and following Wi-Fi exposure: (b) rouleaux 
formations; (c) non-specific clumping of red blood cells; and (d) early fibrin seen as white threads in the blood plasma.
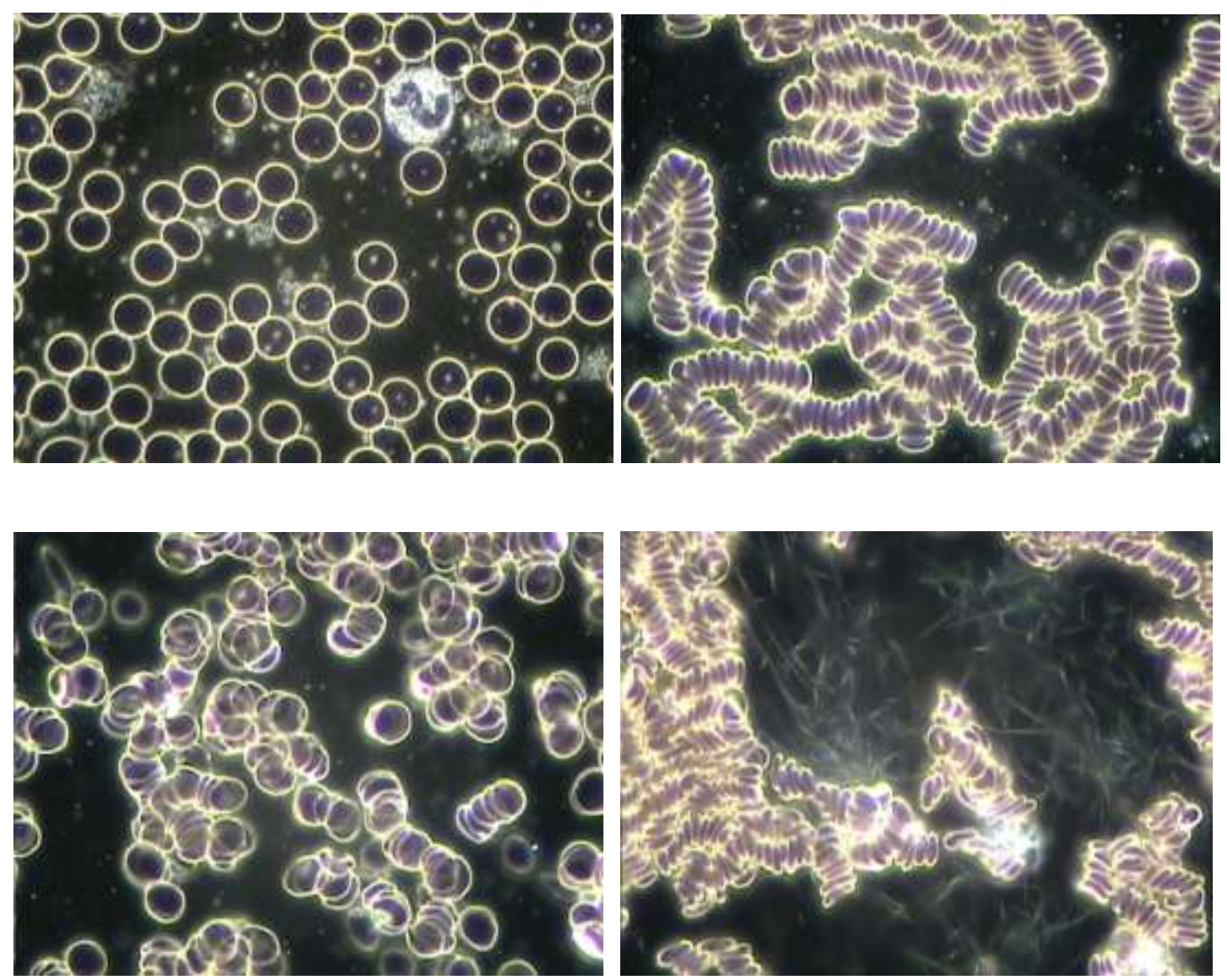

Figure 3. (a), upper left shows healthy blood; (b), upper right shows the formation of rouleaux; (c), lower left shows non-specific RBC clumping; (d) lower right shows early fibrin (white threads) in the blood plasma.

The baseline blood tests of all subjects revealed, for the most part, healthy blood. Immediately following Wi-Fi exposure, all twelve subjects showed statistically significant adverse blood changes: rouleaux formation; non-specific RBC clumping; and occasionally greater quantities of early fibrin and reduced white blood cell motility, as well as occasional 
RBC membrane shape distortions, from round cells to misshapen forms. Figure 4 shows sample blood microphotographs for a female subject, age 66, at (a) baseline, (b) post-exposure, and (c) post-exposure while using the Quantum Bloc.
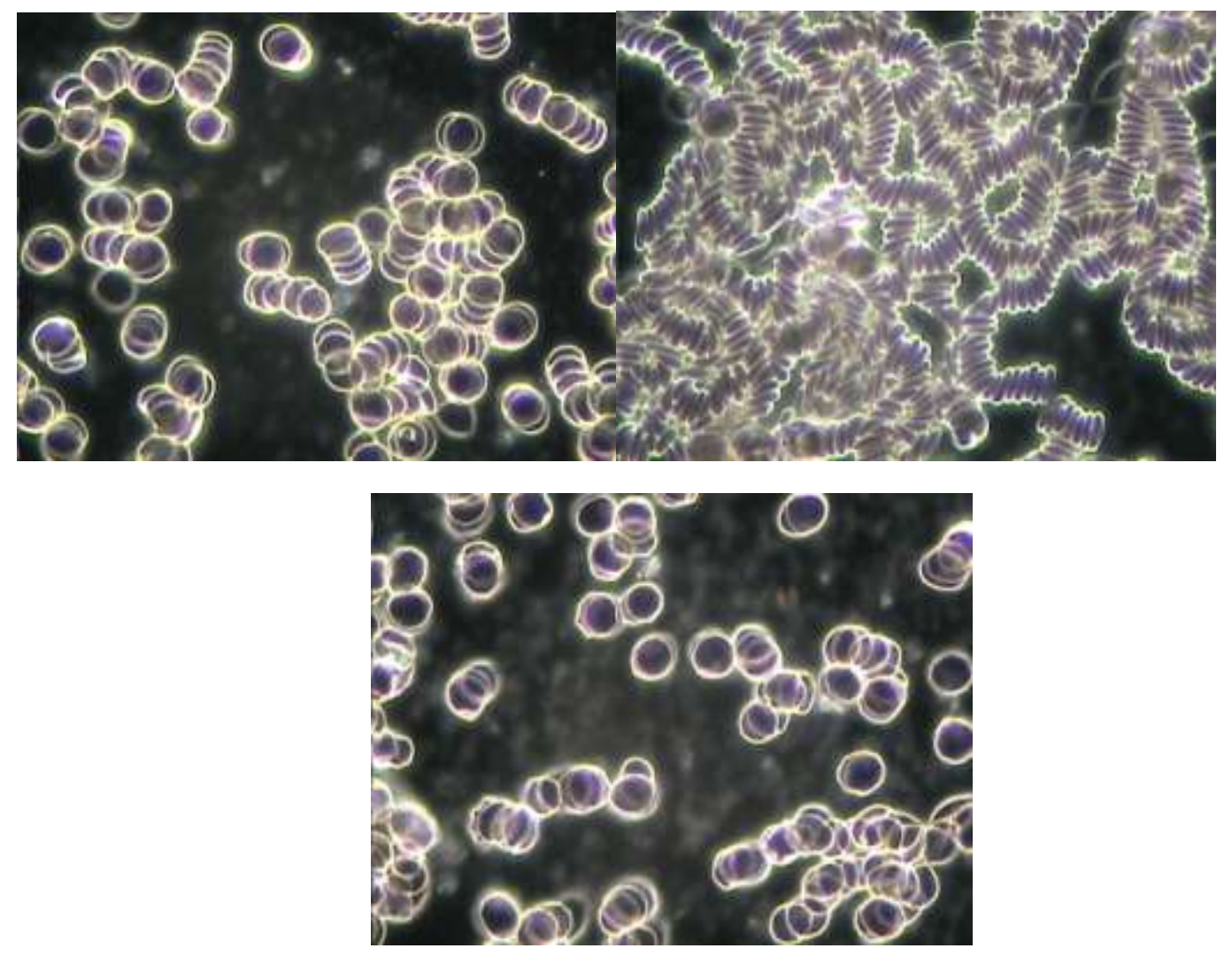

Figure 4. Blood of female subject, 66, at (a) baseline, upper left; (b) post-exposure, upper right; and (c) post-exposure, bottom, with Quantum Bloc.

Using t-tests, no statistical differences were found between the mean values of blood factors for baseline of the sham compared to baseline of the Quantum Bloc, or for the postexposure condition for the sham compared to post-exposure condition of the Quantum Bloc. However, the mean values for baselines and post-exposures showed statistically significant differences for the five blood factors: rouleaux $\left(p=9 \times 10^{-13}\right)$; non-specific aggregates $(p=5 \times$ 
$\left.10^{-12}\right)$; WBC motility $(\mathrm{p}=0.002)$; early fibrin $(\mathrm{p}=0.001)$, and cell membrane distortions (shape changes $)(p=0.003)$. Figure 5 compares the mean relative values of blood factors for the baseline and post-exposure (10 minutes) conditions.

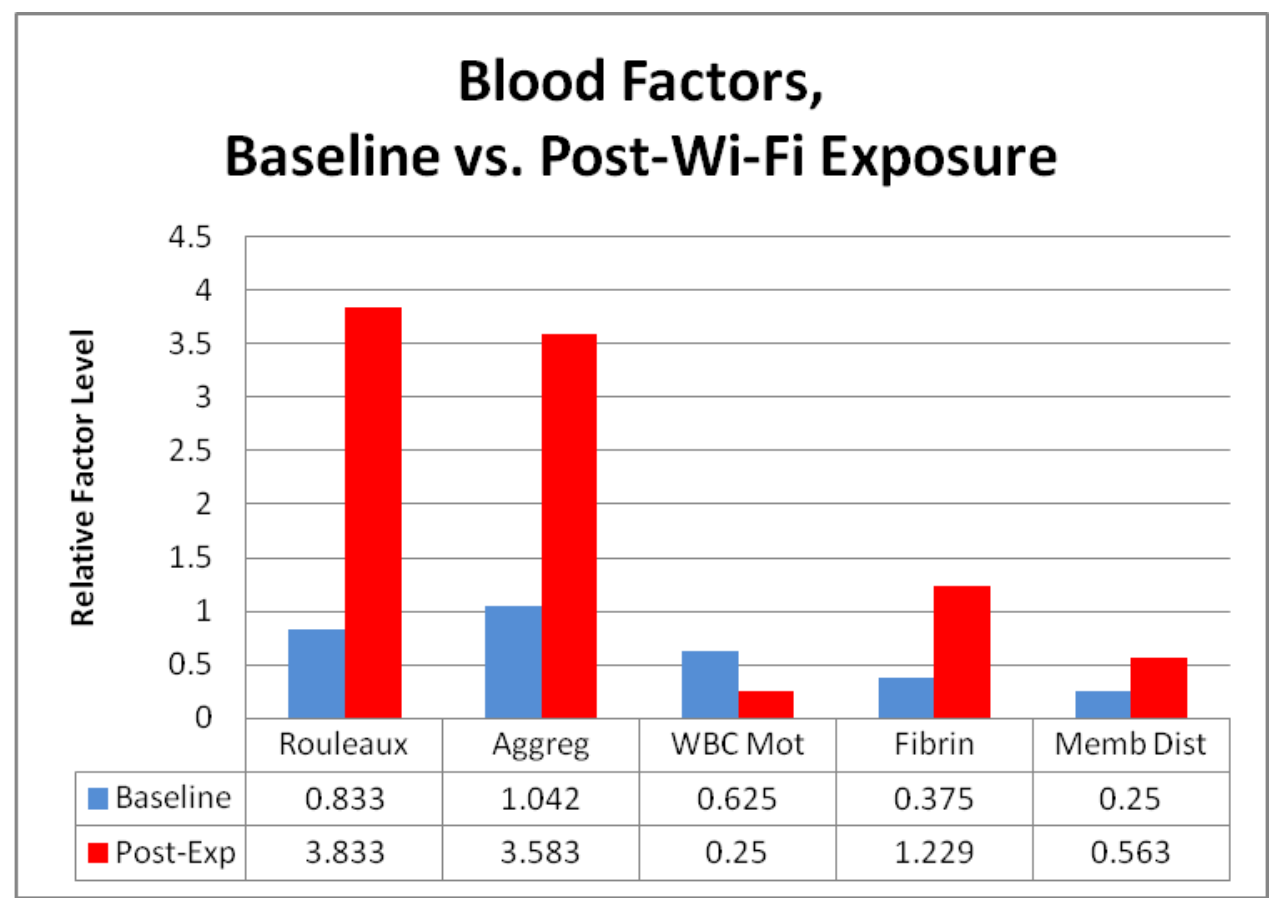

Figure 5. Comparison of blood parameters for baseline (no exposure) vs. post-exposure. Rouleaux = roll formations of red blood cells; Aggreg = generalized non-specific clumping of red blood cells; WBC mot = relative motility (movement) of white blood cells; Fibrin = appearance of early fibrin; and Memb Dist = red blood cell shape changes and distortion

Table 1 shows the mean values of the assessed blood factors for all conditions - baseline, post-exposure, and post-exposure with the devices (sham or Quantum Bloc). P-values were calculated to show whether either device changed the blood factors significantly compared to post-exposure. The blood improved significantly when the sham was used, in terms of reduced rouleaux, non-specific RBC aggregates, reduced fibrin, and WBC motility. Use of the Quantum Bloc showed greater improvements in terms of the mean values of reduced rouleaux, non- 
specific RBC aggregates, reduced fibrin, and reduced membrane disturbances, while it also increased WBC motility. The much smaller p-values obtained for Quantum Bloc indicate much greater statistically significant differences compared to the sham in beneficial effects.

\begin{tabular}{|c|c|c|c|c|c|c|c|c|}
\hline Blood P & meter $\mathbf{M}$ & ean Valu & for Sham vs & Quantu & Bloc & & & \\
\hline & Sham & & & & Quantum & Bloc & & \\
\hline & Baseline & $\begin{array}{l}\text { Post- } \\
\text { exp }\end{array}$ & Post-sham & $\begin{array}{l}P- \\
\text { value }\end{array}$ & Baseline & $\begin{array}{l}\text { Post- } \\
\text { exp }\end{array}$ & Post-QB & P-value \\
\hline Rouleaux & 0.875 & 3.917 & 2.542 & 0.0006 & 0.791 & 3.75 & 0.5 & $3.4 \times 10^{-8}$ \\
\hline Aggreg & 1 & 3.833 & 2.667 & 0.001 & 1.083 & 3.333 & 0.792 & $4.5 \times 10^{-5}$ \\
\hline Fibrin & 0.375 & 1.333 & 0.792 & 0.04 & 0.375 & 1.125 & 0.167 & 0.009 \\
\hline WBC mot & 0.708 & 0.25 & 0.667 & 0.017 & 0.542 & 0.25 & 1.708 & 0.0006 \\
\hline Memb dist & 0.25 & 0.708 & 0.667 & 0.445 & 0.25 & 0.417 & 0.25 & 0.11 \\
\hline
\end{tabular}

Table 1. Mean values of all 12 subjects for baseline (pre-exposure), post-exposure, and post-exposures using devices (sham and Quantum Bloc, QB). Here the p-values test for significant differences between postexposure and post-device condition. Rouleaux = roll formations of red blood cells; Aggreg = generalized nonspecific clumping of red blood cells; WBC mot = relative motility (movement) of white blood cells; Fibrin = appearance of early fibrin; and Memb Dist = red blood cell shapechanges and distortions.

Figure 6 shows how the blood factors change from Wi-Fi exposure to Wi-Fi exposure with use of Quantum Bloc. 


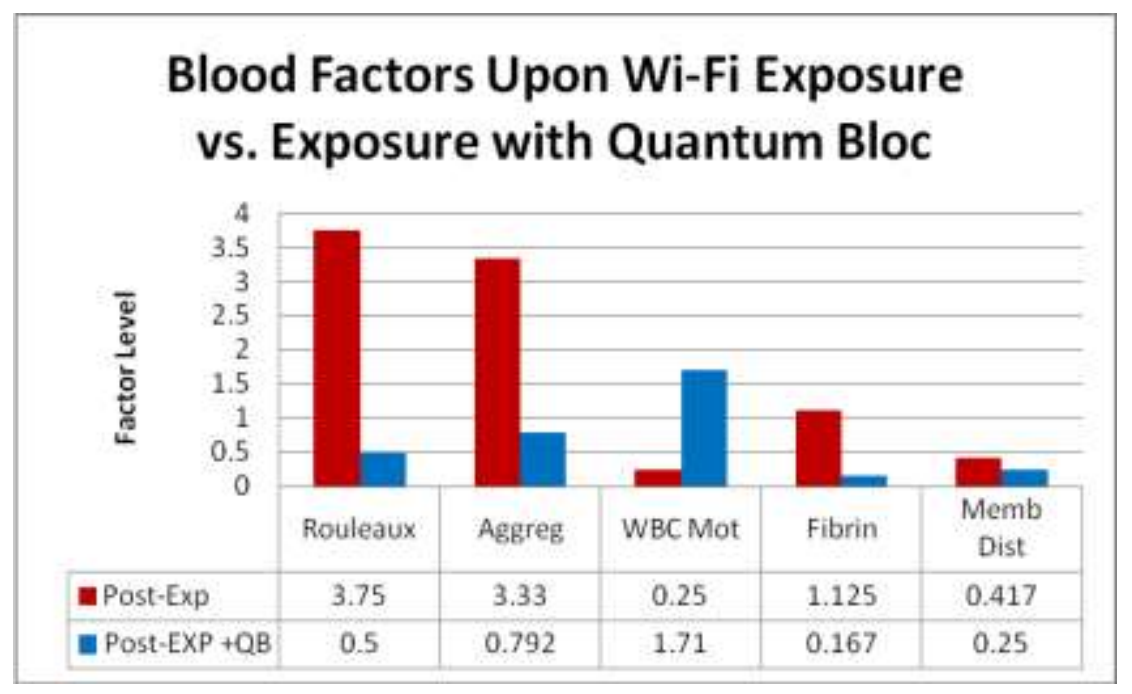

Figure 6. Comparison of blood parameters post-Wi-Fi exposure with Post-Wi-Fi exposure using Quantum Bloc $(\mathrm{QB})$. Rouleaux = roll formations of red blood cells; Aggreg = generalized non-specific clumping of red blood cells; Fibrin = appearance of early fibrin; WBC mot = relative motility (movement) of white blood cells; and Memb Dist $=\mathrm{RBC}$ changes and distortions

The comparison of sham and Quantum Bloc devices for the Wi-Fi exposure condition is shown in Figure 7. This is the critical test of the Quantum Bloc compared to sham. The average values for all 12 subjects in the sham and active device condition are shown. Paired t-tests were done to look for statistical significance between any effects of the sham compared to Quantum Bloc. Significant changes in four blood factors for use of Quantum Bloc compared to sham were found: (1) reduced rouleax formation $(p=0.0004)$; (2) reduced generalized red blood cell aggregation ( $\mathrm{p}=0.001)$; $(3)$ increased white blood cell motility $(\mathrm{p}=0.003)$; and reduced early fibrin $(\mathrm{p}=0.04)$. 


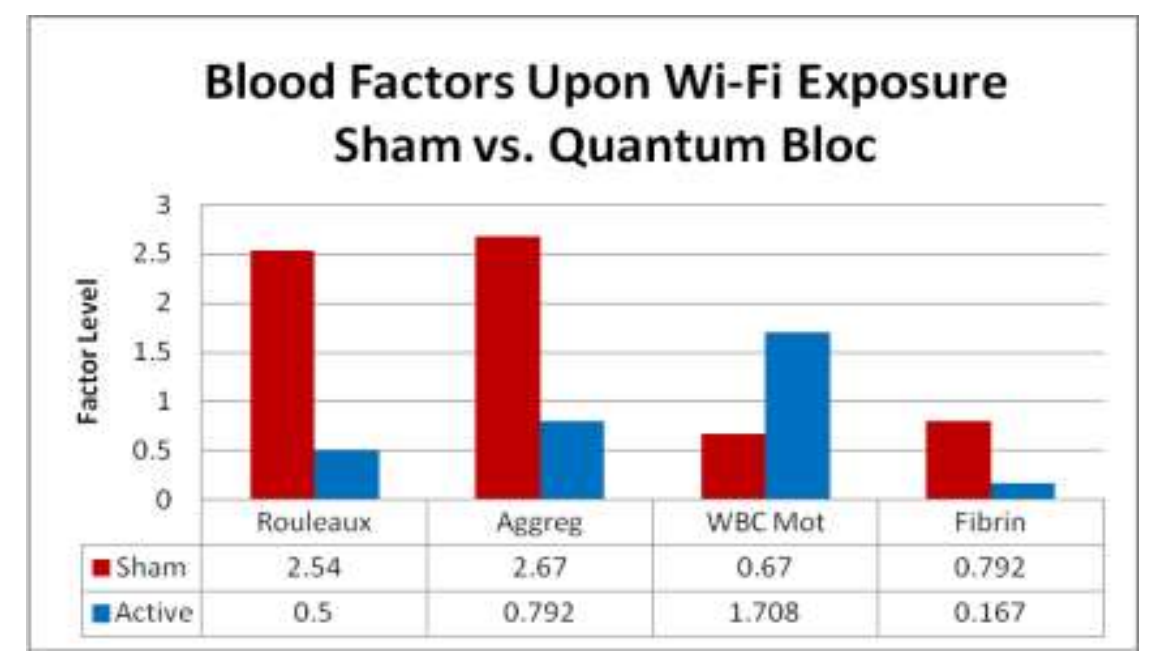

Figure 7: Comparison of blood parameters following Wi-Fi exposure with sham and active device (Quantum Bloc). Rouleaux = roll formations of red blood cells; Aggreg = generalized non-specific clumping of red blood cells; Fibrin = appearance of early fibrin; $\mathrm{WBC}$ mot $=$ relative motility (movement) of white blood cells.

Finally, we tested whether the blood factor values following use of the Quantum Bloc were improved over the baseline values. Table 2 shows this comparison for the mean values of all 12 subjects. Only WBC motility was significantly improved by Quantum Bloc compared to baseline $(\mathrm{p}=0.0018)$; the other changes in parameters were statistically insignificant since $\mathrm{p}>$ 0.05 .

\begin{tabular}{|c|c|c|c|}
\hline \multicolumn{4}{|c|}{$\begin{array}{l}\text { Mean Values of Blood Factors for } \\
\text { Baseline Compared to Quantum Bloc }\end{array}$} \\
\hline & Baseline & QB & P-values \\
\hline Rouleaux & 0.792 & 0.5 & 0.221478 \\
\hline Aggreg & 1.083 & 0.964 & 0.218298 \\
\hline Fibrin & 0.375 & 0.167 & 0.068602 \\
\hline $\begin{array}{l}\text { WBC Mot } \\
\text { Memb }\end{array}$ & 0.542 & 1.708 & 0.001769 \\
\hline Dist & 0.25 & 0.25 & 0.5 \\
\hline
\end{tabular}

Table 2. Mean values for all 12 subjects for baseline (pre-exposure and post-exposures using Quantum Bloc, QB. Here the p-values test for significant differences between baseline and post-device condition. Rouleaux $=$ roll formations of red blood cells; Aggreg = generalized non-specific clumping of red blood cells; WBC mot $=$ relative motility (movement) of white blood cells; Fibrin = appearance of early fibrin; and Memb Dist = red blood cell shape changes and distortions. 


\section{CONCLUSIONS AND DISCUSSION}

Results show readily observable, substantial changes in blood morphology from shortterm exposure to moderate levels of Wi-Fi radiation exposure in all 12 human subjects. RBC aggregations, early fibrin, reduced WBC motility, and RBC membrane shape changes were observed in live blood samples of subjects following 10 minutes of exposure to microwave radiation, and all changes were statistically significant. This confirms that adverse changes in the blood are definitive after 10 minutes of human exposure to $\mathrm{Wi}-\mathrm{Fi}$ from an idle router.

The Quantum Bloc impacted the blood of all 12 subjects positively. Furthermore, it positively impacted all adverse effects of the radiation on the blood that can be readily seen under an optical microscope. Quantum Bloc showed a protective effect over the sham in reducing the formation of aggregates of RBCs and early fibrin formation. This is important because RBC aggregation and early fibrin are involved in blood clotting and are considered risk factors for cardiovascular disease. In addition, Quantum Bloc increased WBC motility compared to sham and baseline values, suggesting a positive influence on the immune response. This could be followed up with more sophisticated immunological studies.

The sham appears to have a small protective effect as well, and most of the mean blood changes of sham with exposure were statistically significant over the post-exposure condition. Such changes may be due to a relaxation effect when sitting with hands extended in the sham device, and/or the placebo effect or "biology of belief," such that a protective effect may result from use of such a device. It is also unclear how long the blood remains disturbed following short-term Wi-Fi exposure. We suspect that the observed blood changes may be reversible. 
Persons exposed may possibly adapt to the stressor over time. However, there is no evidence in the scientific literature of any reversibility, recovery time, or adaptation response. Nonetheless, from the results obtained in this study, it is clear that Quantum Bloc produced more, beneficial, statistically significant changes compared to the sham, indicating a robust protective effect.

This study has important strengths and some limitations. It was a single-blinded, randomized, sham-controlled, microwave exposure-controlled study. Subject fasting was also controlled, as was the time of day when subjects each came to the laboratory for their sessions. An unbiased method of photographing the samples near the center of the blood specimen was used. The researcher has many years of experience in blood microphotography and developed a Likert scale to reliably score blood factors using a well-trained eye. The blood changes recorded by microphotography are clear, objective, and visually compelling.

However, this is a small, short-term study with twelve subjects tested in two experimental sessions. Nonetheless, this study demonstrated a significant protective effect of the Quantum Bloc technology on the blood following only a 10-minute exposure. Larger studies with more subjects, multiple exposures with different or additional EMF devices, and of longer duration of exposure could be done to extend these results. It could also be worthwhile conducting longterm studies in the real world in which humans are exposed to Wi-Fi and other microwave signals throughout the day from numerous communication devices including cell phones, wireless landline phones, computers, tablets, and smart electric meters, to see how the protective effects and benefits from this technology can be best extended and leveraged. 


\section{REFERENCES}

Rubik B. (2014). Does short-term exposure to cell phone radiation affect the blood? Wise Traditions in Food, Farming, and the Healing Arts, Vol 15(4):19- 28.

http://www.westonaprice.org/modern-diseases/does-short-term-exposure-to-cell-phoneradiation-affect-the-blood/ 